\title{
Prolinarylamidase in Humanserum
}

\author{
Von W. Appel \\ Zentrallaboratorium der St.-Vincentius-Krankenhäuser (Professor Dr. med. P. M. Reisert) Karlsruhe und der \\ Kinderklinik Mannheim (Professor Dr. med. E. Huth) der Universität Heidelberg
}

(Eingegangen am 17. August 1981/16. August 1982)

Zusammenfassung: Die katalytischen Konzentrationen einer Prolinarylamidase in Humanserum wurden mittels $L$-Prolin- $\beta$-naphthylamid (Endpunktmethode) und $L$-Prolin-p-nitranilid (kontinuierliche Methode, optimiert) bestimmt. Das Substratoptimum liegt bei $1,53 \mathrm{mmol} / \mathrm{l}$, der $K_{\mathrm{m}}-$ Wert bei $0,13 \mathrm{mmol} / \mathrm{l}$, Substratüberschußhemmung wird nicht beobachtet. Das pH-Optimum liegt bei pH 7,20; Tris-(hydroxylmethyl)-aminomethan- und N-Morpholino-3-propansulfonsäure-Puffer sind gleichwertig. Reduzierende Substanzen, SHReagenzien, Komplexbildner, Antikoagulantien, Proteinaseninhibitoren sowie die Chloride von $\mathrm{Na}^{+}, \mathrm{Cu}^{++}$, $\mathrm{Mn}^{++}$und $\mathrm{Ni}^{++}$besitzen keinen Einfluß. Die schwachen Aktivierungseffekte der Chloride von $\mathrm{Cd}^{++}, \mathrm{Zn}^{++}$, $\mathrm{Hg}^{++}, \mathrm{Co}^{++}, \mathrm{Mg}^{++}$und $\mathrm{Ca}^{++}$sind nicht sicher von Metallproteinatbildungen abzugrenzen. EDTA und Benzethoniumchlorid inhibieren. Serumeigene niedermolekulare Inhibitoren für Prolinarylamidase wurden nicht aufgefunden.

Die katalytischen Konzentrationen liegen in der Regel unter $10 \mathrm{U} / 1\left(37^{\circ} \mathrm{C}\right)$. Die Variationskoeffizienten betragen in Serie um 9,1\%, von Tag zu Tag um 14,5\%. Als vorläufige obere Grenze des Normwertes aus 91 Probanden wird $8 \mathrm{U} / 1$ angenommen. Vergleichende Bestimmungen an Seren von weiteren 372 Patienten mit den $p$-Nitraniliden von Glycin, $L$-Alanin, $L$-Leucin und $\gamma$-Glutaminsäure deuten auf eine diagnostische Sonderstellung der Prolinarylamidase.

Die kontinuierliche Methode konnte für den Vitatron AKES-Analysenayutomaten adaptiert werden.

\section{Proline arylamidase in human serum}

Summary: The catalytic concentrations of proline arylamidase in human sera were determined with $L$-proline- $\beta$-naphthylamide (endpoint-method) and $L$-proline- $p$-nitranilide (continuous reaction). The continuous method was optimized: Tris- $\mathrm{HCl}, 40 \mathrm{mmol} / 1, \mathrm{pH} \mathrm{7,2;}[\mathrm{S}]=1.53 \mathrm{mmol} / \mathrm{l} ; \mathrm{T}=37^{\circ} \mathrm{C}, \mathrm{t}=5-15 \mathrm{~min}$, no additives. The $\mathrm{pH}$-optimum was found to be 7.20; substrate excess inhibition was not observed. Tris-(hydroxymethyl)-aminomethane und $\mathrm{N}$-mōrpholino-3-propane sulfonic acid buffers gave similar reaction rates. Reducing substances, SH-reagents, complex-forming or buffer substances, anticoagulants, proteinase inhibitors and the chlorides of $\mathrm{Na}^{+}, \mathrm{Cu}^{++}, \mathrm{Mn}^{++}$and $\mathrm{Ni}^{++}$did not influence the activity of the enzyme. Slight activation by the chlorides of $\mathrm{Cd}^{++}, \mathrm{Zn}^{++}, \mathrm{Hg}^{++}, \mathrm{Co}^{++}, \mathrm{Mg}^{++}$and $\mathrm{Ca}^{++}$could not be clearly differentiated from the effects of metalloproteinate formation. EDTA and benzethonium chloride inhibited the catalytic activity. In human sera no low molecular weight inhibitors were detectable. Catalytic concentrations were usually in the range below $10 \mathrm{U} / 1\left(37^{\circ} \mathrm{C}\right)$. The coefficients of variation were found to be $9.1 \%$ intraserial and about $14.5 \%$ day-to-day. The preliminary upper limit of "normal" range was established as $8.0 \mathrm{U} / 1\left(37^{\circ} \mathrm{C}\right)$. Comparative simultaneous determinations of the catalytic concentrations, using $L$-leucine-, $L$-alanine-; glycine, $\gamma$ - $L$-glutamic- and $L$-proline-p-nitranilide in the sera of 372 patients, suggest a special diagnostic role for proline arylamidase.

The continuous method has been adapted for Vitatron-AKES-Analyser. 


\section{Einführung}

„Prolinarylamidase“ (EC 3.4.11.2) ist bislang für die klinisch-chemische Diagnostik nicht herangezogen worden. Dieser Begriff steht im folgenden für eine Exopeptidase-Aktivität mit Präferenz für $L$-Prolin$\beta$-naphthylamid oder $L$-Prolin-p-nitranilid.

Der Inhalt der vorliegenden Untersuchung ist die Ausarbeitung und Optimierung einer Routinemethode zur Bestimmung der katalytischen Konzentration dieses Enzyms in Humanserum mit dem Ziel, organ-unspezifische Veränderungen der Konzentrationen bei Erkrankungen mit pathobiochemischen Mechanismen im Bereich des Bindegewebes, des rheumatischen Formenkreises und des Immunsystems sichtbar werden zu lassen.

\section{Material und Methoden}

Geräte

Spektralphotometer Zeiss DM 4 mit Drucker, Küvettenwechselautomatik ZVS 9 und Registrierschreiber Servogor Z 10;

Enzymautomat Eppendorf 5020/II;

Photometer mit Rechner und Drụcker Eppendorf PCP 6121;

Säulenchromatographie-Einrichtung LKB;

Diaflo-Filterkegel (Amicon);

Collodium-Hülsen (Sartorius);

HP-Statistik-Tischrechner 9805 A.

Reagenzien

L-Prolin-p-nitranilid $\left(M_{\mathrm{r}}=235,1\right)$;

$L$-Alanin-p-nitranilid-hydrochlorid $\left(M_{\mathrm{r}}=209,1\right)$;

Glycin-p-nitranilid-hydrobromid $\left(M_{\mathrm{r}}=276,1\right)$ (Fluka, Buchs);

L-Leucin-p-nitranilid $\left(M_{\mathrm{r}}=251,3\right)$;

L- $\gamma$-Glutamyl-3-carboxy-p-nitranilid $\left(M_{\mathrm{r}}=267,1\right)$ (Merck, Darmstadt).

p-Nitranilin, Kalibrierlösung, 0,300 mmol/l (Boehringer Mannheim, Nr. 15912);

Tris-(hydroxymethyl)-aminomethan (Tris), N,N'-bis-(2-Hydroxyethyl)-glycin, N-Morpholino-3-propansulfonsäure (Mops), Ascorbinsäure, N-Ethylmaleinimid, Benzethoniumchlorid, L-Cysteinhydrochlorid, Dithioerythrit, EDTA-di-Natriumsalz, Heparin-Lithium- und -Ammoniumsalz, p-Hydroxybenzoesäure, 8Hydroxylammoniumchlorid, Trypsininhibitor aus Sojabohnen, Zitronensäure-tri-Natriumsalz, Iodacetamid, 1,10-Phenañnthịolin, Phenylmethylsulfonylfluorid (alles Serva, Heidelberg);

$\mathrm{CaCl}_{2}, \mathrm{CdCl}_{2}, \mathrm{CoCl}_{2}, \mathrm{HgCl}_{2}, \mathrm{MgCl}_{2}, \mathrm{MnCl}_{2}, \mathrm{NiCl}_{2}, \mathrm{ZnCl}_{2}$ und $\mathrm{NaCl}$ (alles Merck, Darmstadt);

Aprotinin $\left(\right.$ Trasylol $\left.^{\circledR}\right)=$ Trypsin-Inhibitor aus Rinderorganen (Bayer, Leverkusen);

Sephadex G-25, medium (Pharmacia, Uppsala);

Salzsäurelösung, 1,0 und $0,1 \mathrm{~mol} / 1$, Natronlauge, 1,0 und $0,1 \mathrm{~mol} / \mathrm{l}$, Eisessig, N-Dimethylformamid, Methanol;

tridestilliertes Wasser (Quarzdestillat).

\section{Herstellung der Lösungen}

Kontinuierliche Methode für 80-100 Bestimmungen:

Trispufferlösung, $50 \mathrm{mmol} / \mathrm{l}, \mathrm{pH} 7,20: 0,605 \mathrm{~g}$ Tris in $25,0 \mathrm{ml}$ tridest. Wasser lösen, $45,0 \mathrm{ml} \mathrm{0,1 \textrm {mol } / 1 \mathrm { HCl } - L o ̈ s u n g ~ z u g e b e n , ~}$ auf 100,0 ml auffüllen und pH-Wert mit Glaselektrode prüfen.

Mops-Pufferlösung, $200 \mathrm{mmol} / \mathrm{l}$, pH 6.8: 4,1.80 $\mathrm{g}$ Mops in 100,0 $\mathrm{ml}$ tridest. Wasser lösen und die gewünschten $\mathrm{pH}$-Werte durch Zugabe von 1,0 mol/1 NaOOH-Lösung mittels Magnetrührer und Glaselektrode einstellen.

$\mathrm{NaCl}$-Stammlösung, $5,0 \mathrm{~mol} / \mathrm{l}: 29,2 \mathrm{~g} \mathrm{NaCl}$ in $100,0 \mathrm{ml}$ tridest. Wasser lösen.

Substratlösungen, $20 \mathrm{mmol} / \mathrm{l}: 0,2 \mathrm{mmol}$ des jeweiligen Substrates, z.B. 47,0 mg L-Prolin-p-nitranilid, in 0,2 $\mathrm{ml}$, maximal 0,5 ml Dimethylforamid löșen und auf $10,0 \mathrm{ml}$ mit der jeweiligen Pufferlösung auffüllen.

Herstellung der Lösungen für die Endpunktmethode siehe l.c. (1), für die „Automaten"-Methode siehe Herstellerangaben.

\section{Untersuchungsmaterial}

Die Blutspecimen entstammen stationären Patienten der acht Fachkliniken unserè̃ Krañkenhäuser. Die Blutabnahme erfolgte zwischen 8 und 9 Uhr, die Serumgewinnung anschließend, die Analyse spätestens $3 \mathrm{~h}$ danach.

Hämolytische, ikterische, lipämische und über $3 \mathrm{~h}$ alte Seren s̀owie solche ambulanter Patienten wurden ausgesondert und nicht untersucht.

\section{Bestimmung}

Die Bestimmungsansätze, Reaktions- und Meßbedingưngen sind in Tabelle 1 aufgeführt. Meßwellenlänge $405 \mathrm{~nm}$.

Tạb. 1. Reaktionsbedingungen für die Messung der katalytischen Konzentration der Prolinarylamidase.

$\mathrm{T}=$ Temperatur $\quad \mathrm{d}=$ Schichtdicke

$\mathbf{W}=$ Wellenlänge $\quad \mathbf{A}=$ Absorption
$\mathbf{s}=$ Spaltbreite
$\mathbf{t} \ldots$ Reaktionszeit

\begin{tabular}{lrc}
\hline Lösung & $\mu \mathrm{l}$ & $\begin{array}{l}\text { Konzentration } \\
\text { im Reaktionsansatz }\end{array}$ \\
\hline Trispufferlösuñg pH 7,20 & 1000 & $\begin{array}{c}38,5 \mathrm{mmol} / 1 \\
\text { Substratlösung }\end{array}$ \\
Serumprobe & 100 & $1,53 \mathrm{mmol} / 1$ \\
& 200 & -
\end{tabular}

Kontinuierliche Messung:

$\mathrm{T}=37^{\circ} \mathrm{C} \quad \lambda=405 \mathrm{~nm} \quad \mathrm{~s}=5 \mathrm{~nm} \quad \mathrm{~d}=10 \mathrm{~mm}$

Messung gegen Trispufferlösung

Meßzeit: $\mathrm{t}=5-15 \mathrm{~min}$

Meßbereich: $\Delta=0,05-0,8$

Endpunktsmessung:

$\mathrm{T}=37^{\circ} \mathrm{C} \quad$ nach $\mathrm{t}=3$ und $13 \mathrm{~min}$ je $0,5 \mathrm{ml}$ aliquoten Teil des Reaktionsansatzes der Diàzotierung żựühren.

Mechanisierte Messung:

Vitatron AKES II mit halbierten Volumina und $\mathrm{f}=\mathbf{7 5 4}$

\section{Berechnung}

Die Berechnung der katalytischen Konzentrationen erfolgte bei der Endpunktmethode mit Hilfe einer mit $\ddot{p}$-Nitranilidlösung aufgestellten Bezugskurve, bei der kontinuierlichen Methode über 
die Formel: Katalytische Konzentration $(U / 1)=A \cdot \min ^{-1} \cdot 754$ Die mathematisch-statistische Auswertung wurde mit dem t-Test nach Student für verbundene Stichproben, dem Wilcoxon-Test und dem zweiseitigen Kolmogoroff-Smirnoff-Test mit Hilfe eines Tischrechners und einer EDV-Anlage durchgeführt (siehe auch Legenden zu Tabellen, insb. Tab. 5).

\section{Ergebnisse}

Analytik und Qualitätssicherung bei der kontinuierlichen Bestimmung mittels $L$-Prolin- $p$-nitranilid:

Der Variationskoeffizient in Serie beträgt für $\overline{\mathbf{x}}=$ 5,56 U/1 und $n=18: V_{\mathrm{s}}=9,11 \%$; von Tag zu Tag mit portionsweise eingefrorenen Proben eines Serumpools für $\overline{\mathbf{x}}=12,43 \mathrm{U} / 1$ und $n=8: V_{\mathrm{T}}=$ $14,5 \%$.

Da das Enzym nicht in reiner oder gereinigter Form vorliegt, sind entsprechende Richtigkeitskontrollen durch Zusatz- oder Aufstockversuche nicht möglich.

Die Reaktion verläuft nach einer Vorperiode von 2-3 min über wenigstens $2 \mathrm{~h}$ linear (Abb. 1). Ein-

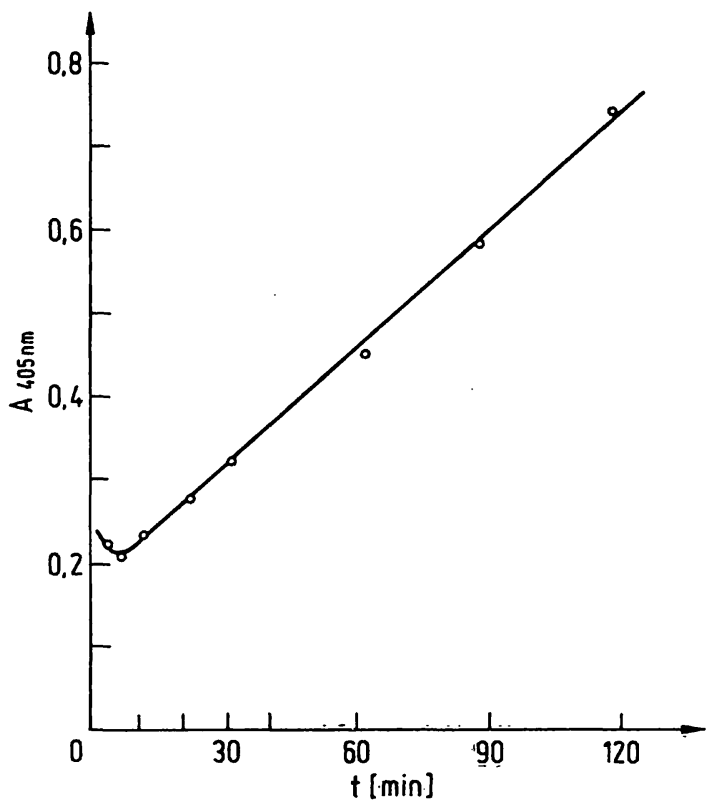

Abb. 1. Prọlinarylamịase in Humanserum. Zeit-Aktivitätsbeziehuing.

Substrat: $\quad L$-Prolin-p-nitranilid, $1,53 \mathrm{mmol} / \mathrm{l}$

Ordinate: Reaktionszeit in min

Abszisșe: Subștratumsatz als A405nm

satz verschiedener Volumina von Seren mit verschiedenen katalytischen Konzentrationen ergibt eine lineare Beziehung (Abb. 2). Eine Substrat-Inhibierung ist bis zu Sättigungskonzentrationen nicht beobachtbar (Abb. 3).

Der $K_{\mathrm{m}}$-Wert beträgt $0,13 \mathrm{mmol} \cdot 1^{-1}$.

Das pH-Optimum der Substrathydrolyse liegt bei pH 7,20; die Kurve nimmt zum saueren Bereich hin

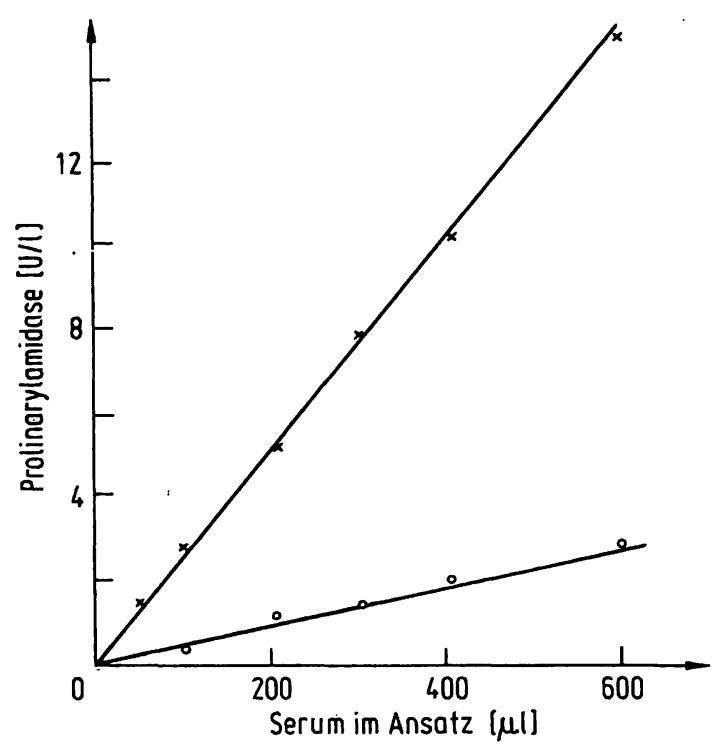

Abb. 2. Prolinarylamidase in Humanserum: Menge-Aktivitätsbeziehung.

Substrat: $L$-Prolin-p-nitranilid, $1,53 \mathrm{mmol} / \mathrm{l}$

Ordinate: Serum im Ansatz in $\mu$ l

Abszisse: Katalytische Konzentration in $U / 1$

O-O Serumpool von Gesunden, $x-x$ Serumpool von Patienten.

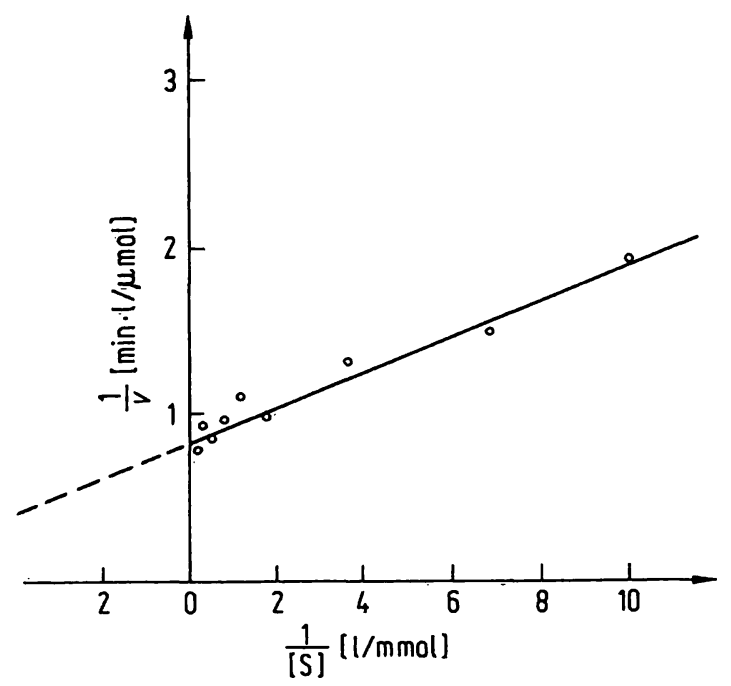

Abb. 3. Prolinarylamidase in Humanserum: Lineweaver-BurkPlot.

Substrat: $\quad L$-Prolin-p-nitranilid

Ordinate: Reziproke Substratkonzentration $1 / S$ in I. $\mathrm{mmol}^{-1}$

Abszisse: Reziproke Reaktionsgeschwindigkeit $1 / v$ in $1 \cdot \mathrm{U}^{-1}=\min \cdot 1 / \mu \mathrm{mol}$

rasch, zum alkalischen hin langsamer ab (Abb. 4). Tris-(hydroxymethyl)-aminomethan- $\mathrm{HCl}$ - und $\mathrm{N}$ Morpholino-3-propansulfonsäure-Na-Puffer verhalten sich gleichartig.

Lagerung von Humanserum bewirkt rasche Inaktivierung. Aufbewahrung nach $5 \mathrm{~h}$ bei $37^{\circ} \mathrm{C}, 17 \mathrm{~h}$ bei Raumtemperatur und $24 \mathrm{~h}$ bei $4{ }^{\circ} \mathrm{C}$ führen zu jeweils $50 \%$ Verlust der katalytischen Konzentrationen. 


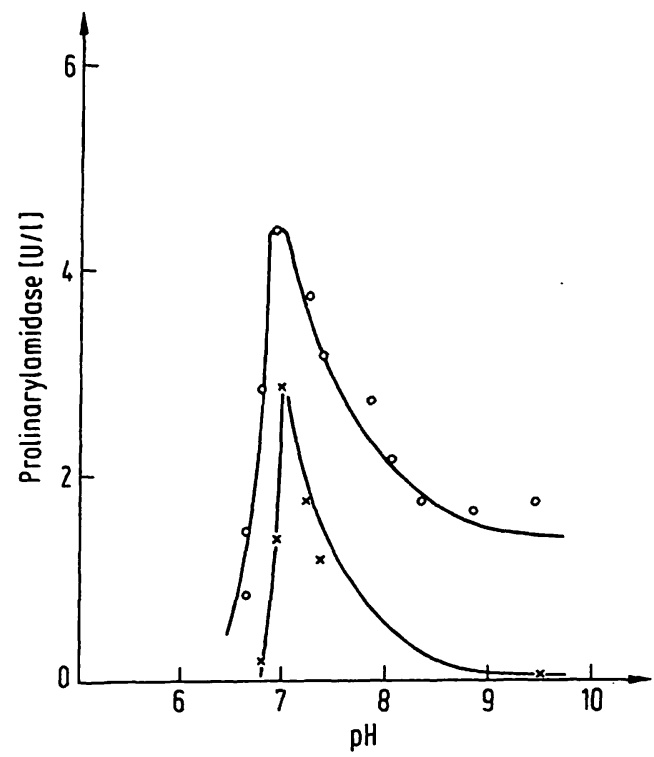

Abb. 4. Prolinarylamidase in Humanserum: pH-Abhängigkeit. Abszisse: pH-Werte

Ordinate: Katalytische Konzentration in U/l $\mathrm{O}-\mathrm{O}$ Tris-(hydroxymethyl)-aminomethan-HCl-Puffer $\mathrm{x}-\mathrm{x}$ N-Morpholino-3-propansulfonsäure-Na-Puffer

Einmaliges Einfrieren und Auftauen ist möglich.

Der Einfluß von Effektoren ist in Tabelle 2 dargestellt. Reduktionsmittel und SH-Reagenzien sind wirkungslos, ebenso Chelat- und Komplexbildner, Gerinnnungshemmer, Puffersubstanzen und Proteinasen-Inhibitoren.

Nicht eindeutig sind die Wirkungen von Metallsalzen. Aus Registrierkurven kinetischer Messungen ergibt sich kein Einfluß der Chloride von $\mathrm{Na}^{+}, \mathrm{Cd}^{++}$, $\mathrm{Co}^{++}, \mathrm{Mn}^{++}$und $\mathrm{Ni}^{++}$dann, wenn diese mit Serum $10 \mathrm{~min}$ bei $37{ }^{\circ} \mathrm{C}$ vor Zugabe des Substrates vorinkubiert werden (Tab. 3a). Wird dagegen mit Serum gestartet, mit und ohne gleichartige Vorinkubation
Tab. 2. Einfluß von Effektoren auf dic katalytische Konzentration der Prolinarylamidase. Keine Vorinkubation.

\begin{tabular}{|c|c|c|}
\hline Effektor & $\begin{array}{l}c \\
{\left[\mathrm{mmol} \cdot \mathrm{I}^{-1}\right]}\end{array}$ & $\begin{array}{l}\text { Rela } \\
\text { tive } \\
\text { Aktivi- } \\
\text { tät }\end{array}$ \\
\hline Serumpoool ohne Zusatz & - & $=1,00$ \\
\hline Ascorbinsäưrẹ & 1,0 & 0,98 \\
\hline $\mathrm{N}$-Ethylmaleinimid & 1,0 & 0,71 \\
\hline Benzethoniumchlorid & 1,0 & $-2)$ \\
\hline$L$-Cystein & 1,0 & 1,18 \\
\hline Dithioerythrit & 1,0 & 0,98 \\
\hline EDTA, di-Natriumsalz & 0,1 & 0,56 \\
\hline Heparin, Ammoniumsalz & 0,05 & $\left.\sim 1,0^{2}\right)$ \\
\hline Heparin, Lithiumsalz & 0,05 & $\left.\sim 1,0^{2}\right)$ \\
\hline p-Hydroxybeñżoesäure & 1,0 & 0,79 \\
\hline 8-Hydroxychinolin & 1,0 & $0 ; 93$ \\
\hline Hydroxylammoniumchlorid & 1,0 & 1,00 \\
\hline Iodacetamid & 1,0 & 0,81 \\
\hline Monoiodacetat & 1,0 & $\sim 1,0$ \\
\hline N, N'-bis-(2-Hydroxyethyl)-glycin & 50 & $\sim 1,0$ \\
\hline N-Morpholino=3-propansulfoñäüure & 50 & $\sim 1,0$ \\
\hline 1,10-Phenanthrolin & 1,0 & 1,06 \\
\hline Phenylmethylsulfonylfluorid & 1,0 & $\left.0,80^{1}\right)$ \\
\hline Aprotinin (Trasylol ${ }^{\circledR}$ ) & $10000 \mathrm{KIE}$ & 1,02 \\
\hline Trypsininhibitor aus Sojabohnen & $1 \mathrm{mg}$ & 0,95 \\
\hline Citronensäure, tri-Natriumsalz & 10 & 1,03 \\
\hline $\mathrm{NaCl}$ & 100 & $\sim 1,0$ \\
\hline $\mathrm{MgCl}_{2}$ & 1,0 & 1,31 \\
\hline $\mathrm{CaCl}_{2}$ & 1,0 & 1,21 \\
\hline $\mathrm{ZnCl}_{2}$ & 1,0 & 1.46 \\
\hline $\mathrm{CdCl}_{2}$ & 1,0 & 1,69 \\
\hline $\mathrm{HgCl}_{2}$ & 1,0 & 1.40 \\
\hline $\mathrm{CoCl}_{2}$ & 1,0 & 1,30 \\
\hline $\mathrm{NiCl}_{2}$ & 1,0 & 1.18 \\
\hline $\mathrm{MnCl}_{2}$ & 1,0 & 1,05 \\
\hline $\mathrm{CuCl}_{2}$ & 1,0 & 1.14 \\
\hline
\end{tabular}

1) Auskristallisierend

$\left.{ }^{2}\right)$ Leichte Trübung bżw. Ausfällung.

Tab. 3a. Metallproteinatbildung: Kinetik der Lichtabsorption (scheinbare Enzymaktivität).

Reaktionsansatz: Standardbedingungen, Konzentration der Metallsalze: stets $0,75 \mathrm{mmol} / 1$, Zahlenwerte: Aus $\Delta A$ A/min bei $405 \mathrm{~nm}$ berechnete scheinbare Enzymaktivitäten in $U / 1$ in den Meßbereichen 2. bis 10. uñ 10. bis 20. Minute. Beim 4. Ansatz zusätzlich berechnet aus $\Delta \mathrm{A} / \mathrm{min}$ bei $(405-620) \mathrm{nm}$.

\begin{tabular}{|c|c|c|c|c|c|c|c|c|c|c|c|}
\hline \multirow{2}{*}{$\begin{array}{l}\text { Start } \\
\text { Vorinkubation } \\
\text { min } \\
\text { Meßwellenlänge } \\
\text { Meßzeiten (min) }\end{array}$} & \multicolumn{2}{|l|}{$\begin{array}{l}\text { Substrat } \\
\text { Serum } \\
10\end{array}$} & \multicolumn{2}{|l|}{$\begin{array}{l}\text { Serum } \\
\text { Substrat } \\
10\end{array}$} & \multicolumn{2}{|c|}{$\begin{array}{l}\text { Serum + Substrat } \\
\text { keine }\end{array}$} & $\begin{array}{l}\text { Serum }-1 \\
\text { keine }\end{array}$ & Substrat & & & \multirow[t]{2}{*}{$\begin{array}{l}\text { Sicht- } \\
\text { bare } \\
\text { Trüu=- } \\
\text { bung }\end{array}$} \\
\hline & $\begin{array}{l}405 \\
2 .-10\end{array}$ & $10 .-20$ & $\begin{array}{l}405 \\
2 .-10 .\end{array}$ & $10 .-20$ & $\begin{array}{l}405 \\
2 .-10\end{array}$ & $10 .-20$ & $\begin{array}{l}405 \\
2 .-10 .\end{array}$ & $10 .-20$ & $\begin{array}{l}405-620 \\
2 .-10\end{array}$ & $10 .-20$ & \\
\hline $\mathrm{NaCl}$ & 3,0 & 1,5 & 1,9 & 1,9 & 3,0 & 2,4 & 2,8 & 2,4 & 2,8 & $2,4 \ldots$ & - \\
\hline $\mathrm{CdCl}_{2}$ & 4,0 & 1,8 & 16,0 & 4,0 & 27,7 & 2,3 & 17,0 & 4,0 & $9 ; 0$ & 1,0 & - \\
\hline $\mathrm{CoCl}_{2}$ & 4,0 & 2,0 & 5,7 & 5,7 & 9,5 & 3,2 & 8,0 & 3,2 & 4,0 & 2,0 & - \\
\hline $\mathrm{MnCl}_{2}$ & 3,0 & 1,4 & 3,2 & 3,2 & 4,4 & 2,7 & 10,0 & 2,6 & 5,0 & 2,0 & - \\
\hline $\mathrm{NiCl}_{2}$ & 1,8 & 1,2 & 17,2 & 4,6 & 3,2 & 2,6 & 9,0 & 3,6 & 4,5 & 2,6 & - \\
\hline $\mathrm{ZnCl}_{2}$ & - & - & - & $=$ & - & - & - & - & - &. & $++t$ \\
\hline
\end{tabular}


mit Substrat, bewirken $\mathrm{Cd}^{++}$und $\mathrm{Co}^{++}$in den ersten 2 bis etwa 10 min starke Absorptionszunahmen, die sich nach 10-20 min Reaktionszeit auf die Werte der Absorptionszunahmen ohne Effektor einpendeln. Durch Zweiwellenlängenmessungen $\Delta \mathrm{A}$ (405)-(620) können unspezifische Trübungseffekte teilweise eliminiert werden (Tab. 3a). Eine Basislinienkorrektur aus Gesamtspektren von 280-700 $\mathrm{nm} \mathrm{zu}$ verschiedenen Reaktionszeiten war nicht möglich, da kein kurzwelliges Minimum der 405 nmBande meßbar ist - ähnlich wie bei den klinischchemischen Routinebestimmungen der Leucinarylamidase und der $\gamma$-Glutamylpeptidase mit $p$-Nitraniliden.

Aus den Gesamtspektren wurde eine Abgrenzung der scheinbaren (durch Metallproteinat-Eintrübung) von den echten (Metallaktivierung) Absorptionszunahmen pro Zeiteinheit gegenüber Ansätzen ohne Metallsalzzusatz versucht. Die (scheinbaren) katalytischen Aktivitäten nach Zusatz von $0,75 \mathrm{mmol} / 1$ $\mathrm{CdCl}_{2}$ lagen in der 10.-20. Minute bei $405 \mathrm{~nm}$ um $4,4 \mathrm{U} / \mathrm{l}$, bei (405-495) $\mathrm{nm}$ um $3,5 \mathrm{U} / 1$ und bei (405-620) $\mathrm{nm}$ bei 1,8 U/1 (gleichzeitiger Start mit Serum und Substrat). In der Anfangsphase der Enzymreaktion bis zu 10 min ergaben sich (scheinbare) Enzymaktivitäten um 15,8, 4,9 bzw. 2,8 U/l. Dieser Effekt nahm bei $\mathrm{Cd}^{++}$-Konzentrationen von 0,025 bis $1,0 \mathrm{mmol} / \mathrm{l}$ stetig zu. Die Metallproteinat- und/ oder Aktivierungseffekte stellen keine Sofortreaktionen dar: Vorinkubationen von $\mathrm{Cd}^{++}$mit Serum, nicht mit Substrat, zeigen die Zeitabhängigkeit dieser Reaktionen (Tab. 3b).

Versuche zur Abtrennung oder Isolierung niedermolekularer Hemmstoffe im Serum mit niedrigen

Tab. 3b. Metallproteinatbildung: Cadmiumchlorid und Prolinarylamidase.

Reaktionsansatz: Standardbedingungen, Konzentration des $\mathrm{CdCl}_{2}: 0,75 \mathrm{mmol} / \mathrm{l}$. Zahlenwertè: Aus den fortlaufendregistrierten Absorptionsänderungen $\Delta \mathrm{A}$ in den MeBbereichen 2. bis 10. und 10. bis 20. Minute in $U / 1$ ausgedrückte tatsächliche (ohne $\mathrm{Cd}^{++}$-Zusatz) oder scheinbare (mit $\mathrm{Cd}^{++}$-Zusatz) Enzymaktivitäten. Zusătzliche Angaben über deren prozentuale Veränderungen.

\begin{tabular}{llrlrlr}
\hline Start & \multicolumn{2}{l}{ Substrat } \\
Vorinkubation & \multicolumn{2}{l}{ Serum mit und ohne $\mathrm{CdCl}_{2}$} \\
Meßwellenlänge & $405 \mathrm{~nm}$ & & & & \\
Meßzeiten (min) & $2 .-10$. & & & & \\
Vorinkubation & ohne & mit & $\%$ & ohne & mit & $\%$ \\
\hline 0 min & 1,3 & 13,0 & 1100 & 1,6 & 1,8 & 113 \\
5 min & 1,5 & 5,0 & 330 & 1,4 & 0,9 & 64 \\
$10 \mathrm{~min}$ & 1,5 & 3,0 & 200 & 1,4 & 0,4 & 28 \\
\hline
\end{tabular}

oder hohen katalytischen Konzentrationen ergaben keine Hinweise auf deren Existenz. Nach Ultrafiltration (Collodium-Hülsen), zentrifugierender Filtration (Diaflo-Filterkegel) und Dialyse gegen verschiedene Pufferlösungen (Rührdialyse bei $4^{\circ} \mathrm{C}$ ) stiegen die katalytischen Konzentrationen im Konzentrat bzw. Innendialysat nicht an. Zusatz eines inaktiven Diffusats zu Innendialysaten, auch solchen mit verringerten Enzymaktivitäten, bewirkten weder Erhöhung noch Verminderung der katalytischen Konzentrationen der Innendialysate. Konzentrierende Ultrafiltration (Amicon-Zellen) bei $4{ }^{\circ} \mathrm{C}$ bewirkte eine zum Konzentrationsfaktor nicht proportionale Erhöhung der Enzymaktivität. Gelfiltrationsuntersuchungen an Sephadex G 25 und G 100 $(1,0 \times 100-\mathrm{cm}$ Säulen) mit tridestilliertem Wasser und Natriumchloridlösungen verschiedener Konzentrationen (stufenweise und als lineare Gradienten) als Äquilibrier- und Elutionsmedium ergaben keine Hinweise auf die Existenz niedermolekularer Enzyminhibitoren.

\section{Untersuchungen an Patientenseren}

Die Messungen wurden aus zeitlichen und technischen Gründen in drei Serien durchgeführt.

Serie I Substratvergleiche: 87-115 Patientenseren.

Endpunktmethode: Aus Gründen besserer Vergleichbarkeit wurde jeweils die gleiche niedrige Substratkonzentration gewählt: für $L$-Prolin- $p$-nitranilid, $L$-Alanin- $p$-nitranilid und Glycin- $p$-nitranilid, $c_{\mathrm{s}}$ $=0,08 \mathrm{mmol} / \mathrm{l}$, für $L$-Leucin- $p$-nitranilid $c_{\mathrm{s}}=4,00$ mmol/l und für $\gamma$-L-Glutamyl- $p$-nitranilid $c_{\mathrm{s}}=3,60$ $\mathrm{mmol} / \mathrm{l}$. Die Werte aller Stichproben weisen eine links-schiefe, nach logarithmischer Transformation eine Gauß'sche Verteilung auf.

Die Wertepaare in den Substratgruppen $L$-Leucin$p$-nitranilid: $L$-Alanin- $p$-nitranilid zeigen eine lineare Regression mit $y=-1,78+0,95 x$ und eine befriedigende Korrelation mit $r=0,81$. Die Paardifferenzen sind im Wilcoxon-Test und - nach logarithmischer Transformation - im t-Test nach Student mit einer Irrtumswahrscheinlichkeit von $\mathrm{p}=0,05$ signifikant (Tab. 4).

In den Substratgruppen $L$-Leucin-p-nitranilid: Glycin- $p$-nitranilid und $L$-Leucin- $p$-nitranilid: $\gamma$ - $L$-Glutamyl-p-nitranilid einerseits, und $L$-Leucin- $p$-nitranilid: $L$-Prolin- $p$-nitrànilid und $\gamma$ - $L$-Glutamyl- $p$-nitranilid: $L$-Prolin- $p$-nitranilid andererseits sind die Werte miteinander nicht korreliert $(r=0,4)$.

Bei einer Einzelauswertung von 91 Seren mit Leucinarylamidaseaktivitäten im Normbereich fallen 12 
Tab. 4. Substratvergleich mit Patientenseren, Serie I (siehe Text). Zeichenerklärung siehe Legende zu Tab. 5.

\begin{tabular}{|c|c|c|c|c|c|c|c|c|c|}
\hline $\begin{array}{l}\text { Substratgruppen } \\
p \text {-Nitroanilide von }\end{array}$ & $\begin{array}{l}\text { Stati } \\
\mathrm{n}\end{array}$ & \multicolumn{2}{|c|}{ Statistische Kenngröße } & $\mathbf{Q}$ & \multicolumn{3}{|c|}{ Regression } & $\begin{array}{l}t-\text { Test } \\
t\end{array}$ & $\begin{array}{l}\text { Wilcoxon } \\
\text { C }\end{array}$ \\
\hline $\begin{array}{l}L \text {-Leucin } \\
L \text {-Alanin }\end{array}$ & $\begin{array}{l}115 \\
115\end{array}$ & $\begin{array}{l}16,8 \\
14,3\end{array}$ & $\begin{array}{l}0,81 \\
0,98\end{array}$ & 0,85 & 0,81 & $-1,78$ & 0,95 & 4,70 & $-5,52$ \\
\hline $\begin{array}{l}L \text {-Leucin } \\
\text { Glycin }\end{array}$ & $\begin{array}{l}114 \\
114\end{array}$ & $\begin{array}{c}17,9 \\
1,09\end{array}$ & $\begin{array}{l}1,39 \\
0,06\end{array}$ & 0,06 & 0,23 & 0,21 & 0,06 & 12,3 & 16.9 \\
\hline $\begin{array}{l}L \text {-Leucin } \\
L \text {-Glutaminsäure }\end{array}$ & $\begin{array}{l}123 \\
123\end{array}$ & $\begin{array}{l}17,3 \\
49,2\end{array}$ & $\begin{array}{l}0,95 \\
4,56\end{array}$ & 2,84 & 0,37 & $-69 ; 8$ & 8,61 & 7,47 & $-7,56$ \\
\hline $\begin{array}{l}L \text {-Leucin } \\
L \text {-Prolin }\end{array}$ & $\begin{array}{l}91 \\
91\end{array}$ & $\begin{array}{c}15,9 \\
0,48\end{array}$ & $\begin{array}{l}0,72 \\
0,08\end{array}$ & 0,30 & 0,07 & 0,20 & $0 ; 01$ & 21,5 & 17,6 \\
\hline $\begin{array}{l}L \text {-Glutaminsäure } \\
L \text {-Prolin }\end{array}$ & $\begin{array}{l}87 \\
87\end{array}$ & $\begin{array}{c}42,4 \\
0,46\end{array}$ & $\begin{array}{l}4,59 \\
1,89\end{array}$ & 0,01 & 0,10 & 0,53 & $-0,01$ & 9,15 & 26,3 \\
\hline
\end{tabular}

Seren mit deutlich bis stark erhöhten Prolinarylamidaseaktivitäten auf; sie verhalten sich jedoch unauffällig gegenüber den vier anderen Substraten.

Serie II Substratvergleiche: 88-127 Patientenseren.

Kontinuierliche Methode: Substratkonzentration für $L$-Prolin-p-nitranilid $c_{\mathrm{s}}=0,14 \mathrm{mmol} / \mathrm{l}$, sonst wie bei Serie I. Die Ergebnisse entsprechen denen der Serie I. Die graphischen Darstellungen (Abb. 5-9) und die statistische Auswertung zeigen wiederum, daß eine Korrelation $(r=0,81)$ nur bei den Wertepaaren der Gruppe $L$-Leucin-p-nitranilid: $L$-Alanin- $p$-nitranilid erkennbar ist (Tab. 5).

Die Einzeldifferenzen können aufgrund des zweiseitigen Kolmogoroff-Smirnoff-Tests mit $\mathrm{p}=0,01$ in der Regel als nicht normal verteilt angesehen werden. Sie (und die Paardifferenżen untereinañder) sind im Wilcoxon-Test für verbundene Stichproben mit $p=0,05$ signifikant. Ihr absoluter Betrag spiegelt die verschiedenen Hydrolysegeschwindigkeiten wider.

Die Einzelbetrachtung in der Gruppe $\dot{L}$-Leucin- $p$ nitranilid: $L$-Prolin- $p$-nitranilid zeeigt (Abb. 8) 11 Seren mit erhöhter Leucinarylamidase, jedoch unauffälliger Prolinarylamidase-Aktivität; umgekehrt weisen 10 Seren hohe hydrolytische Aktivitäten gegenüber $L$-P̈rolin- $p$-nitranilid, jedoch nợmaăle gegenüber $L$-Leucin- $p$-nitranilid auf. Ähnliches gilt für die Gruppe $L$-Glutamyl-p-nitranilid: $L$-Prölin- $p$-nitranilid (Abb. 9).

Tab. 5. Substratvergleich mit Patientenseren, Serie II (siehe Text).

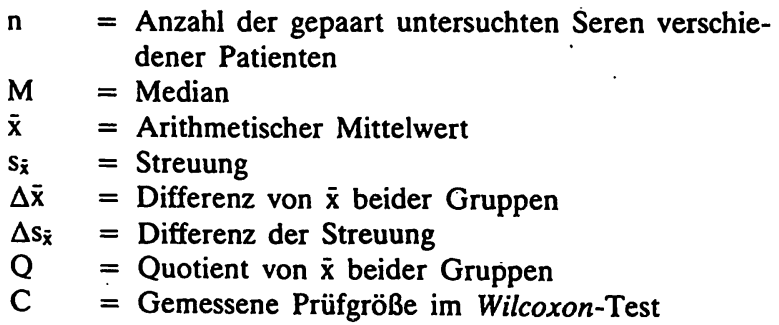
0-Hyp = Null-Hypothese: Die Meßwerte eines Paares von Substraten aus je einem Patientenserumm sind von- einander nicht signifikañt ụnterscheidbar.
$\mathrm{K}=$ Regressionskoeffizient einer linearen Regression
$\mathrm{a}, \mathrm{b} \doteq$ Glieder und Konstanten der linearen Regres- sionsgleichung
$\mathrm{F} \quad=$ Gemessene Prüfgröße in der Varianzanalyse
$\mathrm{t}=$ Gemessene Prüfgröße im gepaarten $\mathrm{t}$-Test nach Student (für Tab. 4).

\begin{tabular}{|c|c|c|c|c|c|c|c|c|c|c|c|c|c|}
\hline \multirow{2}{*}{$\begin{array}{l}\text { Substratgruppen } \\
p \text {-Nitroanilide von }\end{array}$} & \multicolumn{7}{|c|}{ Statistische Kenngrößen } & \multicolumn{2}{|c|}{ Wilcoxon } & \multicolumn{3}{|c|}{ Regression } & \multirow{2}{*}{$\begin{array}{l}\text { Varianz- } \\
\text { Analyse } \\
|F| 0,05\end{array}$} \\
\hline & $\mathrm{n}$ & $\mathbf{M}$ & $\overline{\mathbf{x}}$ & $\mathbf{s}_{\overline{\mathbf{x}}}$ & $\Delta \overline{\mathbf{x}}$ & $\Delta \mathbf{s}_{\overline{\mathbf{x}}}$ & $\mathbf{Q}$ & $\mathrm{C}$ & 0 -Hyp. & $\mathbf{K}$ & a & b & \\
\hline $\begin{array}{l}L \text {-Leucin } \\
L \text {-Alanin }\end{array}$ & $\begin{array}{l}112 \\
112\end{array}$ & $\begin{array}{l}14,55 \\
12,50\end{array}$ & $\begin{array}{l}16,73 \\
14,36\end{array}$ & $\begin{array}{l}0,810 \\
0,953\end{array}$ & 2,366 & 0,517 & 0,86 & $=5,237$ & abgel. & 0,841 & $-2,179$ & 0,988 & 264,5 \\
\hline $\begin{array}{l}L \text {-Leucin } \\
\text { Glycin }\end{array}$ & $\begin{array}{l}110 \\
110\end{array}$ & $\begin{array}{r}14,90 \\
0,92\end{array}$ & $\begin{array}{c}18,05 \\
1,091\end{array}$ & $\begin{array}{l}1,372 \\
0,061\end{array}$ & 16,96 & 1,359 & 0,06 & $-9,267$ & abgel. & 0,227 & 0,910 & 0,010 & 6,1 \\
\hline $\begin{array}{l}L \text {-Leucin } \\
L \text {-Glutaminsäure }\end{array}$ & $\begin{array}{l}127 \\
127\end{array}$ & $\begin{array}{l}14,90 \\
28,90\end{array}$ & $\begin{array}{l}17,31 \\
49,19\end{array}$ & $\begin{array}{l}0,946 \\
4,521\end{array}$ & 31,88 & 4,265 & 2,84 & $-\overline{7}, 857$ & abgel. & 0,367 & 18,83 & 1,573 & $18 ; 81$ \\
\hline $\begin{array}{l}L \text {-Leucin } \\
L \text {-Prolin }\end{array}$ & $\begin{array}{l}90 \\
90\end{array}$ & $\begin{array}{c}14,35 \\
0,310\end{array}$ & $\begin{array}{r}15,84 \\
0,476\end{array}$ & $\begin{array}{l}0,693 \\
0,081\end{array}$ & 15,37 & 0,704 & 0,03 & $-0,238$ & abgel. & 0,079 & 0,622 & $-0,009$ & 0,5 \\
\hline $\begin{array}{l}L \text {-Glutaminsäure } \\
L \text {-Prolin }\end{array}$ & $\begin{array}{l}88 \\
88\end{array}$ & $\begin{array}{c}26,05 \\
0,310\end{array}$ & $\begin{array}{r}42,40 \\
0,449\end{array}$ & $\begin{array}{l}4,597 \\
0,080\end{array}$ & 41,95 & 4,585 & 0,01 & $-8,147$ & abgel. & 0,158 & 0,322 & 0,003 & 2,21 \\
\hline
\end{tabular}



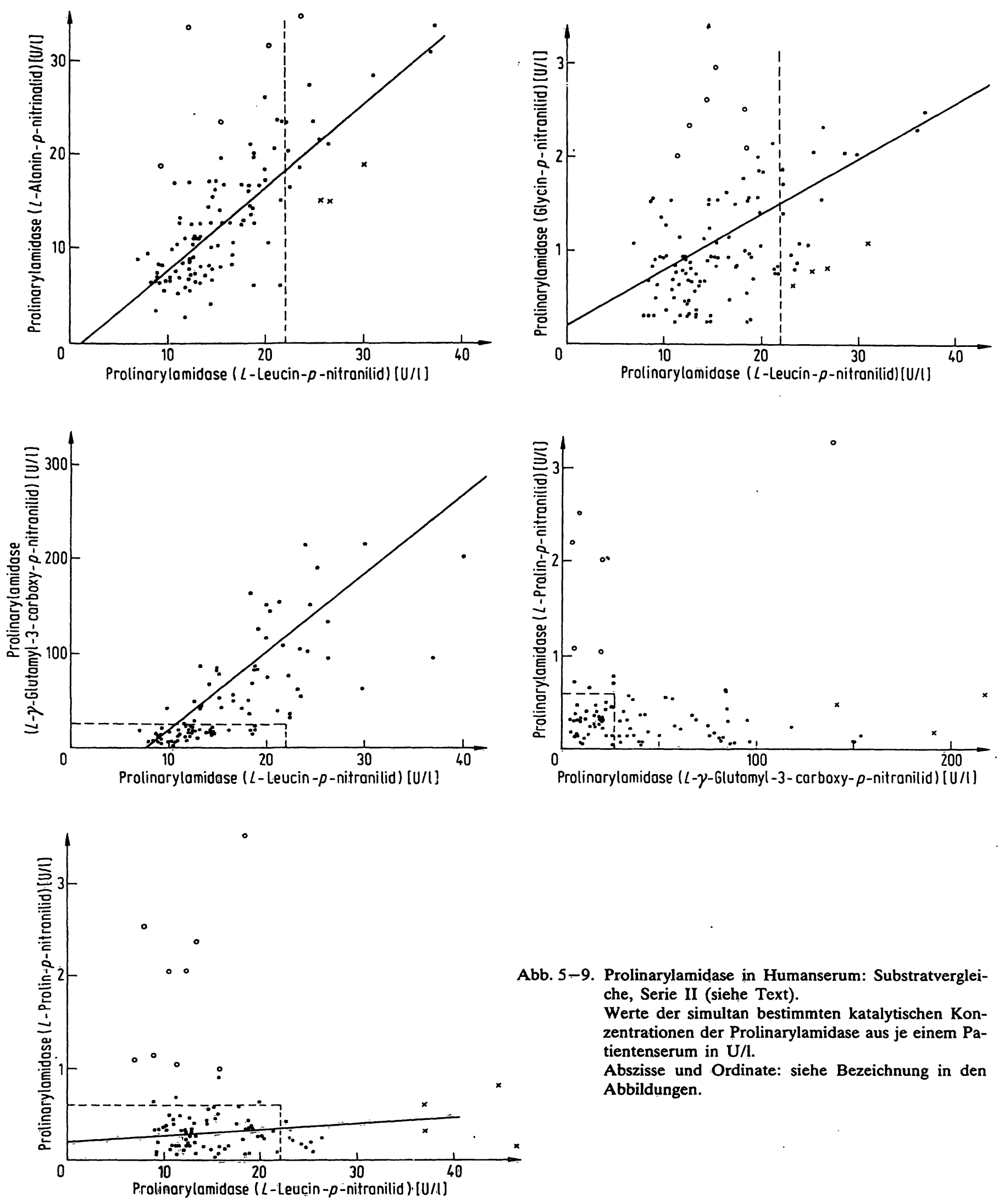

Abb. 5-9. Prolinarylamidase in Humanserum: Substratvergleiche, Serie II (siehe Text).

Werte der simultan bestimmten katalytischen Konzentrationen der Prolinarylamidase aus je einem $\mathrm{Pa}$ tientenserum in $U / l$.

Abszisse und Ordinate: siehe Bezeichnung in den Abbildungen.

Serie III Vorläufiger „Normbereich“ “ für Prolinarylamidase: 130 Patiensenseren, kontinuierliche Methode.

Für das Routineverfahren festgelegte Substratkonzentration für $L$-Prolin-p-nitranilid $c_{s}=1,53$ $\mathrm{mmol} / \mathrm{l}$.
Anhand eines selektierten Patientengutes (vorwiegend Patienten mit banalen oder gastrointestinalen Erkrankungen) wird bei links-schiefer Verteilung ein Median von $M=3,10 \mathrm{U} / \mathrm{l}$ und ein arithmetischer Mittelwert von $\bar{x}=4,86 U / /$ mit einer Streuung von s $=4,70 \mathrm{U} / 1$ gefunden. Nach Elimination der oberen 
Extremwertgrenze (90, 0ter Zentilwert) ergibt sich $\overline{\mathbf{x}}$ $=3,65 \mathrm{U} / \mathrm{l}, \mathrm{s}=1,98 \mathrm{U} / \mathrm{l}$ ein Grenzwert von $\overline{\mathbf{x}}^{\prime}+$ $2.35^{\prime}=8,20 \mathrm{U} / \mathrm{l}$. Somit wird als vorläufiger ,Normbereich": ,,0-8 U/l" festgelegt. Für die patientenbezogene Einzelauswertung werden willkürlich Werte von $8,1 \ldots 14,9 \mathrm{U} / \mathrm{l}$ mit "leicht erhöht $(+)$, von $15,0 \therefore 29,9 \mathrm{U} / \mathrm{l}$ mit erhöht $(++)$ und ' $=$

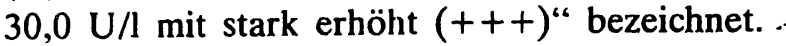

Die Ergebnisse dieser Auswertung und mögliche klinische Gesichtspunkte werden an anderer Stelle mitgeteilt werden.

\section{Diskussion}

Die vorliegende Untersuchung soll einen Beitrag darstellen zur Frage, ob organunspezifische pathobiochemische Reaktionsabläufe im Bereich der Grundsubstanz durch Bestimmung der katalytischen Konzentrationen bestimmter Enzyme im Blutserum faßbar werden. Für die klinisch-chemische Diagnostik, insbesondere im Falle der Longitudinalbeobachtung, sind routinefähige quantitative Analysenverfahren erforderlich. Hierfür eigenen sich für die Bestimmung von Enzymen des Protein- bzw. Peptidabbaus und -umbaus die sog. „chromogenen Substrate" in besonderem Maße. In vorliegendem Falle wird das Prinzip der Hydrolyse von Aminosäurebzw. Peptid-Arylamiden (z.B. bei. $L$-Leucin-p-nitranilid, $L$ - $\gamma$-Glutamyl-p-nitranilid, Tosylglycyl- $L$-propyl- $L$-arginin- $p$-nitranilid) verwendet, um Enzymaktivitäten zu erfassen, die am Kollagenabbau beteiligt sein können. Dabei wird die z.Z. in der klinisch-chemischen Diagnostik heftig geführte Diskussion zur Substratspezifität gegenüber diesem Substrat und dessen Carboxylderivat nicht berührt. Ziel dieser Untersuchung ist die Ausarbeitung eines Verfahrens zur quantitativen Bestimmung der katalytischen Konzentration einer "Prolinarylamidase" mittels $L$-Prolin- $p$-nitranilid unter Routinebedingungen an Patientenseren.

„Arylamidase“ ist ein Ưberbegriff für ein komplexes System von Peptidasen mit überschneidenden Substratspezifitäten, Isoenzymen und multiplen Formen $(1-3)$.

„Prolinarylamidase“ sei die vorläufige Bezeichnung einer Exopeptidase mit Präferenz für $L$-Prolin- $p$-nitranilid und $-\beta$-naphthylamid und ist möglicherweise identisch mit der Dipeptidhydrolase „Prolindipeptidase“ ( $L$-Prolylaminosäurehydrolase, $L$-Prolylglycindipeptidase, Iminodipeptidase, Prolinase, EC 3.4.13.8, früher EC 3.4.3.6).

Dieses Enzym katalysiert die hydrolytische Spaltung der Dipeptidbindung von $L$-Prolylaminosäure in $L$ Prolin und Aminosäure (4-9).
Klassisches Substrat ist $L$-Prolylglycin; hydrolysiert werden $L$-Prolylglycylglycin, Prolyl- $L$-prolin und Salmin, nicht dagegen die entsprechenden $L$-Hydroxyprolinderivate, Aminoaryl- $L$-Prolin („Prolidase"), $L$-Prolyl-L-arginin-, Glycyl- $L$-prolyl- und Glycyl- $L$-prolyl- $L$-alanin-peptide sowije $L$-prolinfreie Di- und Tripeptide (10).

Die Eigenschaften der untersuchten Prolinarylami= dase entsprechen z.T. denen der Prolyldipeptidase, wobei der Hauptunterschied im gegensinnigen Verhalten gegenüber $\mathrm{Co}^{++}, \mathrm{Mn}^{++}$und $\mathrm{Ca}^{++}$liegt. Mikrosomale Arylamidasen werden duch $\mathrm{Co}^{++}$aktiviert $(1,11)$, lysosomale inhibiert $(4)$; bei Sicherung einer $\mathrm{Me}^{++}$-Aktivierung könnte die Prolinarylamidase im Humanserum mikrosomalen Úrsprungs sein. Zugabe von $\mathrm{Zn}^{++}, \mathrm{Cd}^{++}$und $\mathrm{Co}^{++}$, in geringerem $\mathrm{MaBe}$ von $\mathrm{Mn}^{++}, \mathrm{Ca}^{++}$oder $\mathrm{Mg}^{++}$zu Humanserum führt zu einer sofort einsetzenden und zunehmend langsamer verlaufender Eintrübung aufgrund einer Metallproteinatbildung. Diese kann auch durch Messung von Gesamtspektren zu verschiedenen Zeiten einer fortlaufenden Enzymreaktion von echten Aktivierungseffekten nicht eindeutig abgegrenzt werden.

Die Üntersuchung des Arylamidasen-,,Clusters“ im Humanserum deutet auf eine diagnostische Sonderstellung der Prolinarylamidase. Der daraufhin ermittelte vorläufige Entscheidungsbereich für unauffälliges Verhalten wird mit 0-8 U/l angenommen. In einer anschließenden prospektiven Pilot-Studie sollen Erfahrungen zur diagnostischen Sensitivität und Spezifität bei Erkrankungen des rheumatischen Formenkreises, Bindegewebserkrankungen und bei der Strahlentherapie von Tumorerkrankungen gesammelt werden.

\section{Danksagung}

Herrn Professor Dr. Reisert sei an dieser Stelle für die Realisierbarkeit und Unterstützung für diese Studie ausdrücklich gedankt. Dankbare Anerkennung sei Frau Ingrid Kirstein für ihre sorgfältige technische Mitarbeit ausgesprochen. Besonders bedanken möchten wir uns bei Herm Dr. Koller, Abt. Allgemeine Biochemie, Medizinische Forschung der Firma Boehringer Mannnhieim und damit auch dieser Firma für die statistische Auswertung.

\section{Nachtrag}

Inzwischen konnte in einer gezielten klinischen Studie an 90 selektierten Patienten die Sonderstellung der Prolinarylamidase gegenüber carcinoembryonalem Antigen (CEA) und Tennessee polypeptide antigen (TPA) sowie 3 Dipeptidylarrylamidasen gesichert werden. 


\section{Literatur}

1. Appel, W. (1974) Peptidasen. In: Methoden der enzymatischen Analyse, 3. Aufl. (Bergmeyer, H. U., ed.) Verlag Chemie, Weinheim/Bergstraße, Band 1, S. 987ff.

2. Appel, W., Huth, E. \& Hermann, H. (1976) Immunität und Infektion 4, 182-189.

3. Lorentz, K., Marunowski, A. \& Ritter, U. (1974) Z. Klin. Chem. Klin. Biochem. 12, 468-473.

4. Lorentz, K., Koch, C.-D., Flatter, B. \& Molz, J. (1975) Z. Klin. Chem. Klin. Biochem. 13, 45-48.

5. Mäkinen, K. K. (1969) Acta Chem. Scand. 23, 1409-1438.
6. McDonald, J. K., Leibach, F. H., Grindeland, R. E. \& Ellis, S. (1968) J. Biol. Chem. 243, 4143-4150.

7. Oya, H., Nagatsu, T. \& Harada, M. (1970) Experientia 26, 252-253.

8. Unger, Th. \& Struck, H. (1977) Clin. Chim. Acta 78, $113-120$.

9. Hopsu-Havu, V. K. \& Sarimo, S. R. (1967) Hoppe-Seyler's, Z. Physiol. Chem. 348, 1540-1546.

10. Sarid, S., Berger, A. \& Katchalski, E. (1962) J. Biol. Chem. $237,2207-2213$.

Prof. Dr. rer. nat. Walter Appel

Dipl.-Chem., Klin. Chem.

Zentrallaboratorium der St.-Vincentius-Krankenhäuser

Südendstraße 32

D-7500 Karlsruhe 1 